\title{
Simulating the Impact of Cooperation and Management Strategies on Stress and Economic Performance
}

\author{
Josef Daňa \\ Masaryk University, \\ Faculty of Informatics \\ jdana@mail.muni.cz
}

\author{
Ivan Kopeček \\ Masaryk University, \\ Faculty of Informatics \\ kopecek@fi.muni.cz
}

\author{
Radek Ošlejšek \\ Masaryk University, \\ Faculty of Informatics \\ oslejsek@fi.muni.cz
}

\author{
Jaromír Plhák \\ Masaryk University, \\ Faculty of Informatics \\ xplhak@fi.muni.cz
}

\begin{abstract}
In this paper, we study the impact of the management evaluation strategies that are aimed at achieving a balance between rewarding the cooperative behavior of employees and their economic performance. We developed a model in the NetLogo simulation environment that incorporates many socioeconomic aspects such as the stress, effort, and productivity of employees as well as insights into managing cooperativeness and the performance of individual workers. We conducted a series of simulations, each representing a 10-year lifespan of an organization, and the results reveal that organizations achieve the highest performance when management prefers to reward the cooperative behavior of employees instead of performance. The detailed results are provided and discussed in the paper, as are the future directions that the research could take as well as possible extensions of the model presented.
\end{abstract}

Keywords: agent-based modeling, cooperation, organizational performance, Prisoner's dilemma, stress.

\section{Introduction}

Levels of stress and the quality of relationships among co-workers are known to have a direct influence on job satisfaction and physical health, both resulting in a negative economic impact on organizations $[1,2]$.

The role of the management is to establish an optimal working environment that will obviate such problems while aiming to achieve the highest possible organizational performance. As Livio \& De Chiara [3] point out, it is a priori unclear whether the management should either support cooperative or competitive environment between co-workers to promote performance. Some researchers argue that humans are reciprocal by nature [4], and that a friendly environment fosters innovative thinking [5-7], whereas others conclude that competition between co-workers stimulates performance through a higher motivation of individuals $[8,9]$. While the existing studies focus on the interactions within/among the team, and in some exceptional cases on the managerial decisions, our focus is on extending this research by implementing a multi-variable agent-based model. This model allows us to study complex interactions between employees and how the system is influenced by the management strategies that strike a balance between rewarding the cooperative behavior of employees and their direct economic performance for the sake of the profit of the company. The model uses a variety of tunable parameters which define attributes and behavioral rules of agents, whose interaction unfolds in time and create spatial and temporal patterns [10]. The evaluation experiments demonstrated that the model behaves correctly in predictable situations.

The rest of the paper is organized as follows: (1) In Section 2, we locate our approach in the broader context of socioeconomics and game theory. (2) In Section 3, we describe our simulation model, its key features, and principles. (3) In Section 4, we discuss the simulation experiments and their results in detail, and (4) we draw our conclusions and look to the future in Section 5.

\section{Related Work}

According to the American Institute of Stress [11], about three quarters of the U.S. population regularly experience physical and psychological symptoms caused by work related stress and consequent costs to employers are estimated to be $\$ 300$ billion annually. It is now widely accepted that stress has a significant impact on organizational performance as it influences employees' motivation and their sense of wellbeing [2, 12]. The relationship between stress and performance is most commonly explained by the Yerkes-Dodson law [13] where the best performance is achieved at a medium level of stress - the curve describing the relationship is $\cap$-shaped. However, existing field studies on samples of medical nurses [14], software engineers [15], elite football players [16], industrial 
workers [17], surgeons [18], and aviation pilots [19] conclude that there may also be a positive or negative linear relationship between stress and performance. In our model, this relationship is mediated through complex interactions between agents that are based on the prisoner's dilemma game, and further influenced by the management's decision making, as described in Section 3 of this paper.

The prisoner's dilemma game [20], henceforth PDG, is commonly used as a paradigm in studying cooperative behavior. In the original PDG, two players simultaneously decide whether to cooperate or to defect. They receive the reward $R$ or punishment $P$ if both cooperate or defect, respectively. If one player cooperates and the other defects, the former gets a sucker's payoff $S$ while the latter receives a temptation to defect $T$ [21, 22]. In our model, players of PDG correspond to employees that collaborate with their co-workers.

Work by Nowak and May [21] showed that a simple spatial PDG setting can produce remarkably complex and indefinitely changing fractal-like patterns of behavior. Later on, much effort has been devoted to the evolutionary games on different topologies of regular or complex networks [23-29]. Our model is based on a regular lattice where each agent is surrounded by eight neighboring agents (i.e. Moore neighborhood). This basic setting allows us to investigate the dynamics of the cooperation that our model is designed to test, while excluding possible topological effects of interaction. Note that within the context of modeling and simulation, the basic interaction unit in a model is called an agent. In our model, the agent represents an employee, therefore we use words agent and employee interchangeably throughout the text, depending on the context.

Voluntary participation is considered as an effective approach to promote cooperation in PDGs [30-32]. Under this setup, a third strategy called 'loner' is involved. A loner does not temporarily participate in PDG and both, the loner and the other player, take a fixed small payoff. Chen et al. [33] propose an aspiration-induced dormant mechanism, in which loners temporarily quit the game if their payoffs are less than the aspiration level. Our model also permits an agent to temporarily stop participating in the PDG, under the following conditions: agent reaching a given degree of stress become sick, which excludes them from the collaboration for a given number of simulation steps. To mimic real-world situations, the agent returns to work after the "sick leave" ends. However, agents whose recurrent sickness overcomes a given tolerance may quit the job and be subsequently replaced with newcomers.

\section{Model}

Our model represents an organization, such as a company, a university, a factory, etc., where employees are provided with a salary to deliver added value (see section 3.2.1) back to the organization.

The model provides a certain degree of cooperation between individuals - part of the work is done by the employee alone and part of the work can be mutually exchanged with a colleague. This concept is based on the idea that employees exchange the specific work that the other colleague can process more efficiently. This exchange consequently benefits both parties. The willingness to deliver the exchanged part of the work between employees is simulated by PDG. This is a standard approach to studying aspects of cooperation since it is known that spatialized PDG is an undecidable problem [34]. The management of the organization evaluates each employee, where performance and cooperativeness are considered for salary adjustments. The individual workload, the outcome of cooperation, and the salary increase or decrease have an impact on the employees' stress level and consequently on their motivation and related performance.

The core of the model is based on the primary features of cooperation, stress and economic performance. Throughout its development, we have been implementing additional components of the model so that it will be of use to more specific situations. All of the extending components are adjustable by parameters and may be turned off this was mainly used during the testing of the model. While the values of some parameters have to meet certain criteria, e.g. PDG payoff function (see Table 2), the value of other parameters were set arbitrarily, as a result of series of tests. Testing simulations proved that, under given settings, the system is relatively robust and produces reasonable results. Some parameters had to be controlled carefully, as they were highly sensitive to each other (e.g. stress production and stress regeneration) which could significantly influence overall behavior of the system.

\subsection{Terms and Concepts}

In our model, employees are described using the following key features that account for their individual properties.

Strategy is a predefined behavior of the employee when PDG is played. For use in our model, we have selected seven simple strategies commonly used in the PDG context, as listed in Table 1. The strategies that the 
employees use are assigned randomly and never change during their lifespan.

Productivity $\theta$ represents employee's ability to create profit for the company. For the sake of simplicity, we assume each employee having fixed productivity during their lifetime. This number is used in Equation 2 and set as described in Table 3.

Effort $\kappa$ represents an employee's inner motivation to work. While productivity describes the employee's abilities, effort describes how much the employee uses these abilities at a particular moment $-0 \%$ can be understood as "an employee does not work at all" while $100 \%$ as "an employee does their best".

Existing research addressing the influence of external rewards on employees' performance and motivation comes to contradictory conclusions - some state that pay increases the employee performance [35], while others found payment harmful to intrinsic motivation [36]. Cameron et al. [37] pointed out that the influence is also affected by the attractiveness of tasks. In our simplified model, the only factor affecting the effort are changes in salary. Decreasing the salary demotivates employees and decreases their effort and vice versa. However, the influence is not linear. Employees with a high current effort are more sensitive to a salary decrease which leads to a noticeable decrease in their effort. Conversely, employees with a low current effort react more significantly to a salary increase.

Resistance to stress is modeled as a capacity of a virtual stress container. If the stress level is below the given threshold, the employee is healthy and working. When the stress level exceeds the limit, the employee becomes sick and unable to work. When a sick employee returns to work, the stress container is emptied. See Subsection 3.2.2 for closer details.

Sickness is a metaphor used to simulate the impact of high stress on working activities of employees. Sick employees do not produce any profit, but the company still supports them financially. Employees that are sick too often are fired and replaced. The tolerance for repeated sick leave is adjustable in the model, common to all employees, and fixed during the simulation.

\subsection{Simulation Step of One Employee}

Each simulation round can be considered as one working day during which an employee produces a certain amount of added value. In what follows, we explain how this amount is calculated and how it is influenced by certain features of employees, their willingness to cooperate, and stress factors.

3.2.1. Fulfilling the Work Tasks: The primary input for this step is a salary $\beta$, which represents the amount of money that the employee is given by the management.

The management expects that the employee will produce the work at least commensurate with the value of the salary. The primary output is an added value $V$, which corresponds to the input salary increased (or decreased) as a result of the work produced. The added value is affected by three weighted components $V_{e}, V_{c}$, and $V_{r c}$ :

$$
V=\beta\left(V_{e}+V_{c}+V_{r c}\right) .
$$

Efficiency contribution $V_{e}$ reflects the employee's current working effort $\kappa$ relative to their productivity $\theta$. Cooperation weight, $w_{v}, 0 \leq w_{v} \leq 1$ denotes the portion of the work which is mutually exchanged between two employees, while the rest is done directly by one employee:

$$
V_{e}=\left(1-w_{v}\right) \theta \kappa
$$

Cooperation contribution $V_{c}$ reflects the cooperation initiated by the employee:

$$
V_{c}=w_{v} \phi
$$

This contribution can be interpreted as exchanging the same portion of the work with a co-worker because they are able to do it more effectively and vice versa.

$\phi$ is the results of the iterated PDG played with a co-worker. The game is driven by the payoff matrix defined in Table 2 . The values satisfy condition $T>$ $R>P>S$ and can be interpreted as follows:

- Sucker's payoff $S$ : I did the partner's work as was agreed, but my partner did not do my work. I cannot earn anything from my work because it was not done.

- Cheater's payoff $T$ : I did not do the partner's work, but the partner did my work as we agreed. Because they did the work more efficiently than me, I get the payoff 1.25. Moreover, I had time to do my own work, and so the final payoff is 2.25 .

- Punishment $P$ : We did not agree on cooperation and did the work as usual, with normal efficiency.

- Reward $R$ : Both of us met the agreement, and because we did the work more efficiently, we get a payoff 1.25 . 


\begin{tabular}{lcl}
\hline Strategy & Label & Description of behavior. \\
\hline Defect & D & Always defects. \\
Cooperate & C & Always cooperates. \\
Tit for Tat & T & Repeats partner's last move. \\
Tit for two Tats & T2 & $\begin{array}{l}\text { Defects only if defected in the last two rounds, otherwise cooperates. } \\
\text { Tit for Tat — Naïve Peacemaker }\end{array}$ \\
nT & $\begin{array}{l}\text { Repeats partner's last move. When defected in the last round, there is a } \\
\text { probability of cooperative response. }\end{array}$ \\
Pavlov & P & $\begin{array}{l}\text { Starts with cooperation. Repeats action when won last round and switches } \\
\text { action when lost last round. }\end{array}$ \\
Unforgiving & U & $\begin{array}{l}\text { Once defected by a partner, it will always respond with a defection. } \\
\text { Otherwise cooperates. }\end{array}$ \\
\hline
\end{tabular}

Table 1. The description of employees' collaboration strategies.

In each simulation round, an employee can cooperate with a different co-worker. The collaboration history of the particular partner is always taken into account, which is important for strategies that decide their move based on previous ones, e.g., the "Tit for Tat" strategies, see Table 1 .

\begin{tabular}{lcc}
\hline & Cooperate & Defect \\
\hline Cooperate & $R=1.25$ & $S=0$ \\
Defect & $T=2.25$ & $P=1$ \\
\hline
\end{tabular}

Table 2. The payoff matrix of the PD game.

Requested cooperations contribution $V_{r c}$ : Apart from the cooperation initiated by the employee, any of the neighbors can also ask them for cooperation. Because the cooperation is interpreted as an exchange of part of the work, the requested cooperations $V_{r c}$ have a similar effect to the added value as before, but seen now from the opposite direction:

$$
V_{r c}=\sum_{j} w_{j} \phi_{j}
$$

where $j$ corresponds to the $j$-th requested cooperation, $w_{j}$ is the cooperation weight assigned to the co-worker (co-worker's $w_{v}$ ), and $\phi_{j}$ is the employee's results of the iterated PDG played with the $j$-th co-worker.

3.2.2. Stress Factors: Fulfilling the tasks is stressful. We deal with two types of stress factors, namely, inter-individual and intra-individual stress. The impact of both types of stress on employees is adjustable.

Inter-individual stress is associated with the collaboration $\phi$ and its extent depends on the result of the PDG for every simulation step.
Intra-individual stress results from the subjective perception of the employee. The stress level of every employee is updated relative to their current effort $\kappa$. The more effort an employee exerts, the more stress is produced (regardless of productivity).

Stress regeneration is a mechanism incorporated into every simulation step to counterbalance stress production. The value of regeneration is adjustable by the model.

Resistance to stress is conceived as a capacity to withstand a certain amount of stress. If the total increased stress is greater than the value of regeneration, the stress level rises further. Once it exceeds the capacity of the "stress container", the employee becomes sick. For the stress container, we assume a normal statistical distribution among the population, similar to other personality traits that are determined by numerous independent factors.

\subsection{Lattice of Employees}

The employees are arranged in a square lattice, as illustrated in Figure 1. At the beginning of each simulation, the collaboration strategies of employees, their productivity and stress limits are set up to meet the desired parameters.

In the beginning, the company earmarks a budget $\alpha \in \mathbb{N}$ for all employees. This budget may remain the same for the whole simulation or it can be slightly adjusted at every step, depending on the economic performance of the employees. Then the simulation algorithm performs the Simulation step of an employee as discussed in Section 3.2 to calculate their values added $V_{i}$. The steps follow these rules:

- All employees have exactly eight neighbors. This also applies to the employees at the edge of the interaction plane, as the end of any row or any 

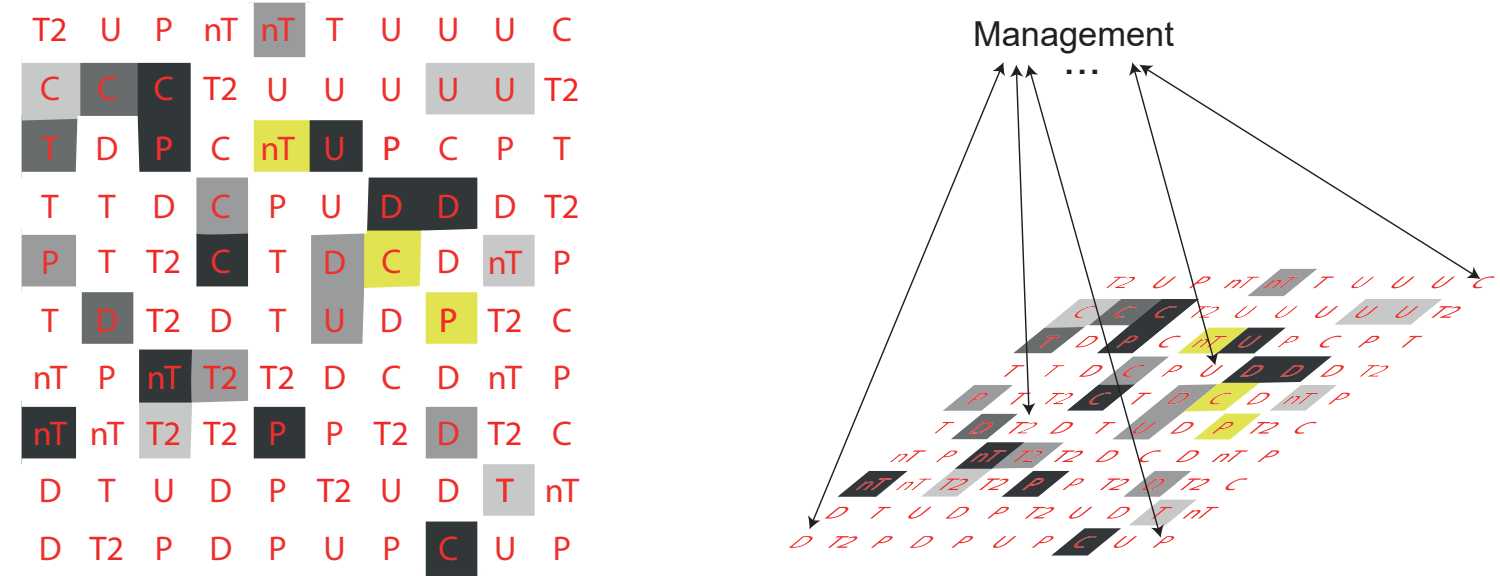

Figure 1. The visualization of the model. Each square represents an employee and their strategy (see Label in Table 1). The gray color scale indicates stress level where darker gray refers to a higher level of stress, and the yellow color implies that the given employee is sick.

column continues from the beginning of the same row or column. The interaction place appears to be a square but is in fact a torus (see Figure 1).

- The employees interact with their neighbors only. Therefore, the $V_{r c}$ contribution in Equation 4 is calculated from at most eight co-workers.

- A neighbor for the interaction is chosen randomly. Sick co-workers are omitted, and their added value $V_{i}$ is set to zero. Also, if there is no healthy neighbor available for the cooperation, then the added value $V_{i}$ of the employee is set to zero.

- The salary $\beta_{i}$ given to the $i$-th employee is calculated from the distribution of the budget $\alpha$ among all employees as follows:

$$
\beta_{i}=\frac{\alpha \rho_{i}}{n}
$$

where $n$ is the number of employees in the lattice. The performance $\rho_{i}$ represents a relative efficiency of the employee compared to other employees. Its value is set to 1 by default for all employees, which ensures an even distribution of the budget among them. However, the management can influence this value based on the results of an employee's evaluation in a way that prospective employees can be motivated by higher salaries (see Equation 9 in Section 3.4).

The final profit generated by each employee is calculated as a difference between their added value and input salary: $V_{i}-\beta_{i}$. The total of profits generated by all employees during one simulation round, in a chosen period, or continuously from the beginning of a simulation, are observed during the simulation to analyze the impact of the factors on the profit of the company.

Apart from the inter-individual and intra-individual stress factors discussed above, changes in salary $\beta_{i}$ represent an additional factor that affects stress. The increase of the salary decreases the employee's stress and vice versa. The amount of stress caused by salary change is modified by the employee's stress capacity - the greater the capacity, the higher the stress level is increased or decreased. Moreover, the current salary is taken into consideration during the stress calculation. The lower the salary, the more stressful any further reduction is, and conversely, a lower stress regeneration is associated with any salary increase.

\subsection{Management Insight and Intervention}

3.4.1. Evaluation of Employees Behavior: After a certain number of simulation steps, given by the parameter evaluation period, the management evaluates employees' behavior taking into account two aspects: cooperativeness and effort. The evaluation $E_{i}$ of $i$-th employee is computed by the relation:

$$
E_{i}=w_{e} C_{i}+\left(1-w_{e}\right) F_{i},
$$

where $C_{i}$ and $F_{i}$ are a cooperation-based insight and an effort-based insight respectively, and $0 \leq w_{e} \leq 1$ 
is a weight, which enables us to prioritize $C_{i}$ and $F_{i}$. Later in the paper, this weight is denoted as management strategy.

Cooperative employees are beneficial for the company. To reflect this phenomenon, the cooperation-based insight $C_{i}$ is used by the management to privilege cooperating employees:

$$
C_{i}=w_{c} \frac{c_{i}}{c_{i}+d_{i}}+\left(1-w_{c}\right) \epsilon,
$$

where $c_{i}$ is the number of cooperations that the employee participated in during the management evaluation period of one month, $d_{i}$ is the number of defections, and $0 \leq \epsilon \leq 1$ is a random number. The weight $0 \leq w_{c} \leq 1$ reflects how much insight the management has into the cooperativeness of employees: 0 means random decision (no insight at all) while 1 means that the management has precise information about the cooperativeness of the employees. Employees that are sick during the whole inspected period have no cooperation so only a random number $\epsilon$ is used for them.

In a similar manner, the management can use insights into the employees' effort. The effort-based insight $F_{i}$ is adjustable by the weight parameter $0 \leq$ $w_{f} \leq 1$, where 0 means 'random decision' (no insight) and 1 means that precise information about the effort of employees is available. The effort-based insight is defined as follows:

$$
F_{i}=w_{f} \frac{\sum W_{i}}{\max _{1 \leq j \leq n}\left(\sum W_{j}\right)}+\left(1-w_{f}\right) \epsilon,
$$

where $\sum W_{i}$ is the added value that the $i$-th employee accumulated during the inspected period, $n$ is the number of employees, and $0 \leq \epsilon \leq 1$ is a random number.

For healthy employees, $W_{i}=V_{i}$ and $F_{i}$ privileges employees working harder than others, as expected. However, sick employees produce $V_{i}=0$ which would result in a penalization in the same way as healthy employees are penalized for their zero effort. To prevent this, $W_{i}$ of sick employees are set to their salary $\beta_{i}$ so that they are not directly penalized for being sick.

3.4.2. Impact on Salary: The result of an evaluation (see Equation 5) is involved in the salary distribution process. After every evaluation, the performance parameter $\rho_{i}$ is re-calculated, taking into account the evaluation result $E_{i}$ of the i-th employee compared with the average evaluation value $E_{\text {avg }}$ of all employees:

$$
\rho_{i}=\rho_{i}+\rho_{i}\left(\frac{E_{i}}{E_{a v g}}-1\right) w_{\rho},
$$

where $0 \leq w_{\rho} \leq 1$ is a parameter that defines the intensity of the impact of the evaluation process on employees' performance.

\subsubsection{Impact of employees' substitution:} Management interventions based on the evaluation process influence the stress of individual employees and, consequently, their sickness. Repeatedly sick employees leave the organization and are substituted. The model also incorporates the substitution of employees that have a low performance over an extended period. The tolerance for sickness and low performance are adjustable and are common for all employees. This mechanism of substituting employees can be seen either as firing an employee due to their long-term poor performance or that employees left the company because of frequent sickness caused by non-cooperating co-workers or bad decisions of the management which do not reflect the employee's effort sufficiently.

Unlimited substitution of employees would be unrealistic because the number of available workers on the job market is limited, depending on the unemployment rate. Furthermore, hiring new employees usually brings additional costs to the organization as newcomers require training and have reduced performance during this period. To bring our model closer to reality, we incorporated a penalization for each employee being substituted by a newcomer. This penalization reduces a company's profit and corresponds to additional costs related to the hiring process and the training of new employees. The amount of penalization is adjustable by a parameter and also reflects the average salary of employees in the organization.

\section{Testing and Simulations}

Our model has been implemented and evaluated in the NetLogo [38] simulation environment. For the repository with the source code, see [39]. Each simulation included 3,600 simulation steps. Taking each step as one day, the results of the simulations can be seen as covering a period of ten years in the life of an organization that is providing monthly evaluation of employees with the consequent intervention of the management. The values of the fixed parameters used for the simulations are summarized in Table 3. For any given simulation setting, 100 runs were executed to obtain average values and eliminate possible bias caused by any stochasticity of the simulation process.

The simulations covered the impact of management 


\begin{tabular}{lcll}
\hline Features of employees & & \\
$w_{v}$ cooperation weight & $20 \%$ & The portion of assigned work to be done with a co-worker \\
$\kappa$ & effort & $75 \%$ & Initial effort of all employees \\
$\theta$ & productivity & $2.8 \pm 0.1$ & Mean value and standard deviation of the distribution \\
& stress limit & $70 \pm 5$ & Mean value and standard deviation of the distribution \\
\multicolumn{2}{c}{ cooperativeness } & $4: 1$ & Proportion of employees with cooperative vs. defective strategies \\
\hline $\begin{array}{l}\text { Features of the management } \\
\text { evaluation period }\end{array}$ & 30 steps & Frequency of the management intervention \\
\multicolumn{2}{l}{ patience } & 12 & Tolerance for repeated sicknesses or low-performance of the employees \\
\hline
\end{tabular}

Table 3. Fixed settings of simulations.

strategies on the economic performance of an organization. These results are presented in the following section.

\subsection{Impact of Management Strategies on Profit}

The two 3D scatter plots in Figure 2 provide an overview of the impact of the management interventions on the company's profit. The horizontal axes represent the insights that the management has into employees' cooperation (insight into cooperation $=w_{c}$ in the model) and performance (insight into performance $=$ $w_{f}$ in the model) respectively, where $0=$ no insight, $1=$ absolute insight. The vertical axes encode the management evaluation strategy $w_{e}$ from 0 (only the performance of employees is taken into consideration) to 1 (only the cooperativeness of employees is taken into consideration), see section 3.4.1. The color of the individual samples encodes the simulation results, as depicted in the color bar on the right-hand side of the figure. The darker hue of red indicates that a higher profit has been generated by the organization at the given settings.

A detailed analysis and visualization of the data lead to the following observations:

- Focusing on cooperativeness and a sufficiently deep insight into the cooperation result in the highest value produced by an organization.

- The strategy based mostly on cooperativeness $\left(w_{e}>0.8\right)$ can achieve an approximately $80-90 \%$ performance level compared to the best result, as long as the insight for cooperation is also high enough (i.e. $w_{c}>0.8$ ). In such settings, the impact of performance insights is weak, and its particular value has no significant influence on the total value of an organization.

- The lowest observed performance (below 10\% compared to the best result) is achieved by an organization where the management has very low or zero insight into both cooperation and performance regardless of which aspect of the work is preferred. Such a setting creates a favorable environment for agents with defective strategies whose population fluctuate around $30-40 \%$ of the total agent population, compared to the initial $20 \%$.

- Balanced strategies $\left(w_{e}=0.5\right)$ can achieve a fair relative performance (i.e. $60-70 \%$ ) only if the insight for cooperation is relatively high $\left(w_{c}>\right.$ 0.7 ). When $w_{c}$ drops below 0.5 , an organizations performance does not exceed $50 \%$ of the best result.

- Strategies that prefer to reward individual performance $\left(w_{e}<0.2\right)$ are performing poorly. The results show that it is still better to reflect cooperativeness at least a bit, as the company can achieve $25-40 \%$ of the best performance as long as the insight for cooperation is high $\left(w_{c}>0.6\right)$. A management strategy that completely disregards cooperativeness of agents $\left(w_{e}=0\right)$ can never perform better than $23 \%$ of the best result for $w_{f}=0.6$ while higher values of $w_{f}$ result in an even lower performance. Having precise information about individual performance $\left(w_{f}=1\right)$ accounts for only approximately $13 \%$ of the best performance. In other words, even when the management rewards agents completely on their performance, it is reasonable to tolerate fluctuation in an agent's performance which is achieved by a submaximal insight for performance.

\subsection{Impact of Management Strategies on Stress}

The scatter plot in Figure 3 encodes the impact of management insight and the evaluation strategy 

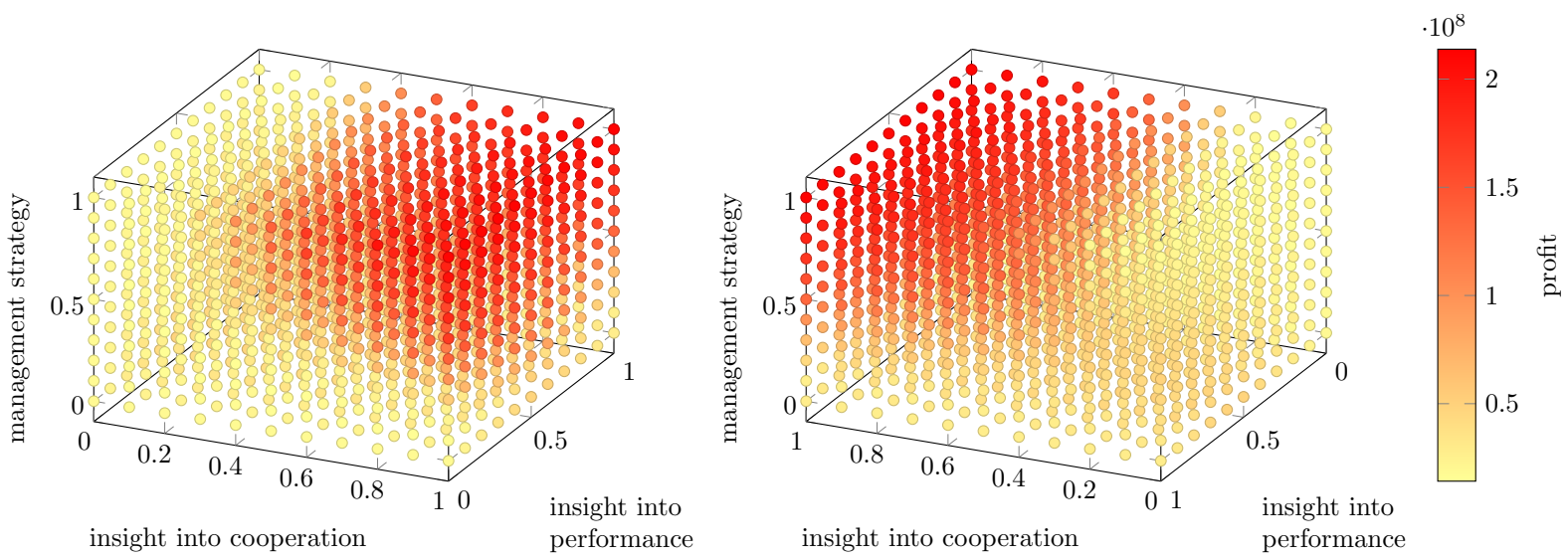

Figure 2. The impact on profit of the management's insight and strategy.
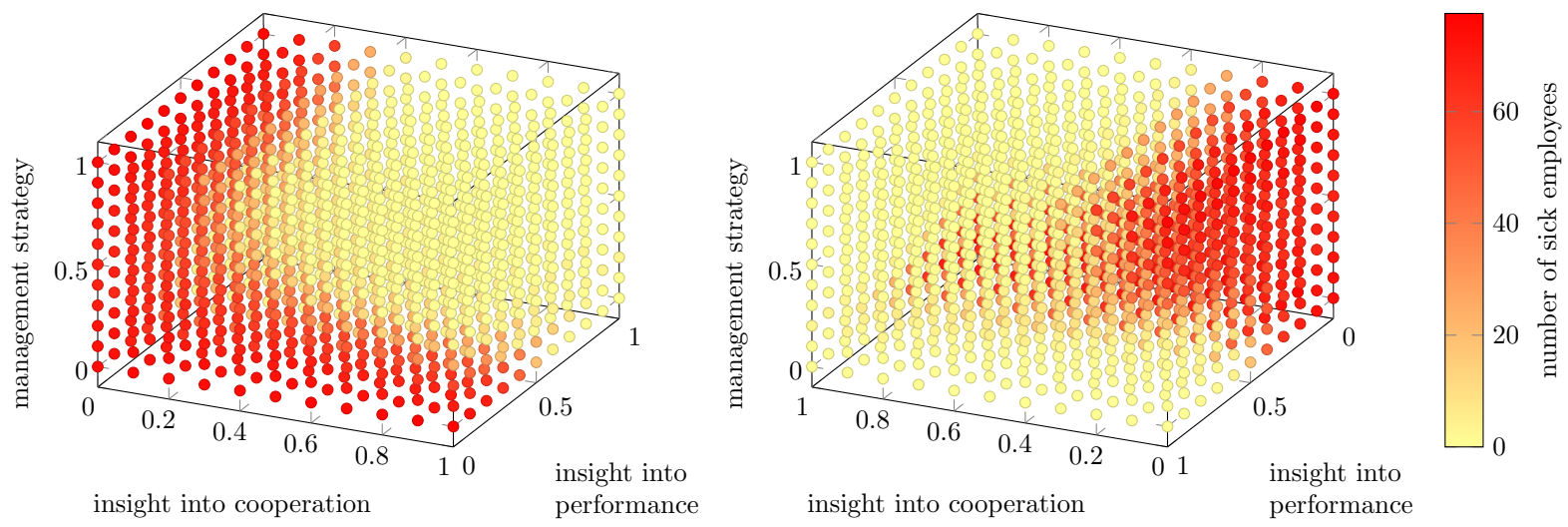

Figure 3. The impact on sickness of the management insight and strategy.

on the total number of employees that have left the organization due to frequent sickness. As sickness correlates with stress, we can interpret these results also as the impact on stress.

The rate of sickness is high when the management strategy is focused on rewarding performance while the insight for performance is low, or when the strategy aims at rewarding cooperation and the insight for cooperation is low. In other words, the two above-mentioned rewarding strategies exhibit high levels of randomness, resulting in a high level of stress among employees. See Figure 3 for details.

\subsection{Impact of Management Strategies on Employment Fluctuation}

Figure 4 depicts the impact of management interventions on the fluctuation of employees. The fluctuation is calculated as a total count of employees that leave the organization, either due to repeated sickness or to long-term underperformance.

As illustrated in Figure 4, a strategy of rewards that aims to improve performance causes high fluctuation when the insight for performance is also high.

\section{Conclusions and Future Work}

We have developed a multi-variable model based on a prisoner's dilemma game in the NetLogo simulation environment. This model allows us to study various aspects of cooperation among employees in an organization. The key parameters are organizational performance, employment fluctuation resulting from stress level, sickness rates and the individual performance of employees. We have also implemented a concept of management strategy which represents a decision how to reward employees' cooperative behavior and individual performance. This concept is extended by implementing a management insight, i.e. a parameter describing the accuracy of information that the management applies when employees are rewarded. Less than maximal insight can be perceived as a level of tolerance for rewarding or punishing employees' behavior. 

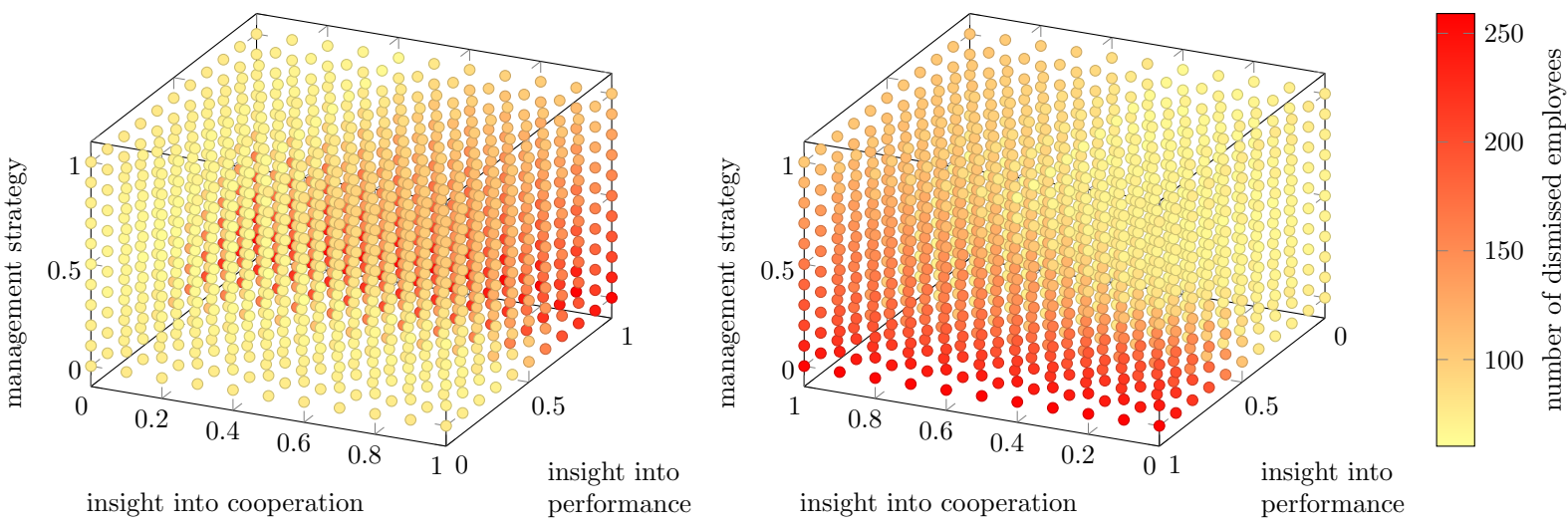

Figure 4. The impact of the fluctuation of employees on the management's insight and strategy.

Our experiments have shown that management strategies and the quality of insight into employees' cooperativeness and performance has a significant effect on both the organizational performance and the employees' wellbeing. The highest organizational performance is achievable in settings when the management focuses on rewarding the cooperativeness of employees. In comparison, focusing solely on individual performance accounts for an organizational performance approximately four times lower. The lowest observed performance (less than $10 \%$ of the highest result) is related to the situation when employees are rewarded randomly. This situation also reports the highest levels of stress. The employment fluctuation reaches its peak when management prefers to reward individual performance and have precise information about it, therefore there is no tolerance for a suboptimal performance of employees.

The experiments demonstrate the possibilities of our model at their current state of development and provide results that are general in nature. To obtain specific results on which conclusions and recommendations for an organization can be formulated, the parameters of the model need to mirror the real situation in a particular company. This can be a starting point for a case study in future research.

The model can be further extended by implementing a social network model where the interaction of employees would be more complex, influenced by the topology of a network, and exploring the potential impact of more central agents on the overall performance of the organization.

There are several other suggestions for future versions of the model, such as the introduction of organizational growth to reflect the economic prosperity of the company, implementing a more realistic model of employees' productivity, involving personality types to fit specific organizations, and to focus on the space and distance between employees that may have an impact on the quality of interactions.

Finally, the employment fluctuation algorithm can be further developed. It should be possible to relocate the employees or change their collaboration strategy before they leave the organization. And a higher penalization of a company for repeatedly dismissing employees could be implemented to better simulate a situation where there is low unemployment in the job market.

\section{References}

[1] S. E. Sullivan and R. S. Bhagat, "Organizational stress, job satisfaction and job performance: Where do we go from here?," Journal of Management, vol. 18, no. 2, pp. 357-374, 1992.

[2] K. Pugliesi, "The consequences of emotional labor: Effects on work stress, job satisfaction, and well-being," Motivation and Emotion, vol. 23, no. 2, pp. 125-154, 1999.

[3] L. Livio and A. D. Chiara, "Friends or foes? optimal incentives for reciprocal agents.," Journal of Economic Behavior \& Organization, 2018.

[4] D. H. Thomas Dohmen, Armin Falk and U. Sunde, "Homo reciprocans: Survey evidence on behavioural outcomes.," The Economic Journal, vol. 119, p. 592 612, 2009.

[5] J. Chen, W. S. Leung, and K. P. Evans, "Are employee-friendly workplaces conducive to innovation?," Journal of Corporate Finance, vol. 40, pp. $61-79,2016$.

[6] X. Tian and T. Y. Wang, "Tolerance for failure and corporate innovation.," The Review of Financial Studies, vol. 27, p. $211255,2011$.

[7] Y. W. Seo and S. W. Chae, "Market dynamics and innovation management on performance in smes: Multi-agent simulation approach," Procedia Computer Science, vol. 91, pp. 707 - 714, 2016.

[8] B. Beersma, J. R. Hollenbeck, S. E. Humphrey, H. Moon, D. E. Conlon, and D. R. Ilgen, "Cooperation, competition, and team performance: Toward a 
contingency approach.," Academy of Management Journal, vol. 46, p. 572 590, 2003.

[9] J. Luft, "Cooperation and competition among employees: Experimental evidence on the role of management control systems.," Management Accounting Research, vol. 31, pp. $75-85,2016$.

[10] N. Nan, "Capturing bottom-up information technology use processes: A complex adaptive systems model," MIS Quarterly, vol. 35, pp. 505 - 532, 2011.

[11] "The American Institute of Stress: stress statistics." https://www.stress.org/stress-research/. Accessed: 2018-05-28.

[12] J. W. Ness, V. Tepe, and D. R. Ritzer, The science and simulation of human performance. The address: Emerald Group Publishing Limited, 2004.

[13] R. M. Yerkes and J. D. Dodson, "The relation of strength of stimulus to rapidity of habit-formation.," Journal of Comparative Neurology and Psychology, vol. 18, p. 459 482, 1908.

[14] S. J. Motowidlo, J. S. Packard, and M. R. Manning, "Occupational stress: its causes and consequences for job performance.," Journal of applied psychology, vol. 71, p. 618 629, 1986.

[15] M. Laanti, "Agile and wellbeing-stress, empowerment, and performance in scrum and kanban teams.," IEEE 46th Hawaii International Conference on System Sciences (HICSS), p. 4761 4770, 2013.

[16] D. A. Savage and B. Torgler, "Nerves of steel? stress, work performance and elite athletes.," Applied economics, vol. 44, p. 2423 2435, 2012.

[17] F. Zhong, E. Yano, Y. Lan, M. Wang, Z. Wang, and X. Wang, "Mental ability and psychological work performance in chinese workers.," Industrial Health, vol. 44, p. 598 603, 2006.

[18] C. M. Wetzel, R. L. Kneebone, M. Woloshynowych, D. Nestel, K. Moorthy, J. Kidd, and A. Darzi, "The effects of stress on surgical performance.," The American Journal of Surgery, vol. 191, p. 5 10, 2006.

[19] S. J. Vine, L. Uiga, A. Lavric, L. J. Moore, K. Tsaneva-Atanasova, and M. R. Wilson, "Individual reactions to stress predict performance during a critical aviation incident.," Anxiety, Stress \& Coping, vol. 28, p. 467 477, 2015 .

[20] R. Axelrod and W. D. Hamilton, "The evolution of cooperation," science, vol. 211, no. 4489, pp. 1390-1396, 1981.

[21] M. A. Nowak and R. M. May, "Evolutionary games and spatial chaos," Nature, vol. 359, no. 6398, p. 826, 1992.

[22] M. Perc and P. Grigolini, "Collective behavior and evolutionary games - an introduction," Chaos, Solitons \& Fractals, vol. 56, pp. $1-5,2013$. Collective Behavior and Evolutionary Games.

[23] Z.-X. Wu and Y.-H. Wang, "Cooperation enhanced by the difference between interaction and learning neighborhoods for evolutionary spatial prisoner's dilemma games," Phys. Rev. E, vol. 75, p. 041114, Apr 2007.

[24] G. Szabó and A. Szolnoki, "Cooperation in spatial prisoner's dilemma with two types of players for increasing number of neighbors," Phys. Rev. E, vol. 79, p. 016106, Jan 2009.
[25] M. c. v. Perc, A. Szolnoki, and G. Szabó, "Restricted connections among distinguished players support cooperation," Phys. Rev. E, vol. 78, p. 066101, Dec 2008.

[26] Z. Rong and Z.-X. Wu, "Effect of the degree correlation in public goods game on scale-free networks," $E P L$ (Europhysics Letters), vol. 87, no. 3, p. 30001, 2009.

[27] A. Szolnoki, M. Perc, and G. Szabó, "Diversity of reproduction rate supports cooperation in the prisoner's dilemma game on complex networks," The European Physical Journal B, vol. 61, pp. 505-509, Feb 2008.

[28] H.-X. Yang, W.-X. Wang, Z.-X. Wu, Y.-C. Lai, and B.-H. Wang, "Diversity-optimized cooperation on complex networks," Phys. Rev. E, vol. 79, p. 056107, May 2009.

[29] S. Ding, J. Wang, S. Ruan, and C. Xia, "Inferring to individual diversity promotes the cooperation in the spatial prisoner's dilemma game," Chaos, Solitons \& Fractals, vol. 71, pp. 91 - 99, 2015.

[30] G. Szabó and C. Hauert, "Evolutionary prisoner's dilemma games with voluntary participation," Physical Review E, vol. 66, no. 6, p. 062903, 2002.

[31] C.-L. Chen, X.-B. Cao, W.-B. Du, and Z.-H. Rong, "Evolutionary prisoners dilemma game with voluntary, participation on regular lattices and scale-free networks," Physics Procedia, vol. 3, no. 5, pp. 1845 - 1852, 2010. The International Conference on Complexity and Interdisciplinary Sciences. The 3rd China-Europe Summer School on Complexity Sciences.

[32] D. Jia, C. Shen, H. Guo, C. Chu, J. Lu, and L. Shi, "The impact of loners' participation willingness on cooperation in voluntary prisoner's dilemma," Chaos, Solitons \& Fractals, vol. 108, pp. 218 - 223, 2018.

[33] Y.-S. Chen, H.-X. Yang, and W.-Z. Guo, "Aspiration-induced dormancy promotes cooperation in the spatial prisoner's dilemma games," Physica A: Statistical Mechanics and its Applications, vol. 469, pp. $625-630,2017$.

[34] P. Grim, "The undecidability of the spatialized prisoner's dilemma.," Theory and Decision, vol. 42, pp. 53 - 80, 1997.

[35] D. G. Gardner, L. Van Dyne, and J. L. Pierce, "The effects of pay level on organization-based self-esteem and performance: A field study," Journal of Occupational and Organizational Psychology, vol. 77, no. 3, pp. 307-322, 2004.

[36] E. L. Deci, R. Koestner, and R. M. Ryan, "A meta-analytic review of experiments examining the effects of extrinsic rewards on intrinsic motivation.," Psychological bulletin, vol. 125, no. 6, p. 627, 1999.

[37] J. Cameron, K. M. Banko, and W. D. Pierce, "Pervasive negative effects of rewards on intrinsic motivation: The myth continues," The Behavior Analyst, vol. 24, no. 1, pp. 1-44, 2001.

[38] U. Wilensky, "Netlogo," tech. rep., Northwestern University, Evanston, IL, 1999.

[39] J. Daňa, I. Kopeček, R. Ošlejšek, and J. Plhák, "Git repository with the model." https://gitlab.fi.muni.cz/lsd/PDG-cooperation-simulator.git, 2018. 